\title{
Developing an Ontology-Based Cold Chain Logistics Monitoring and Decision System
}

\author{
Yujun Wang, Jianjun Yi, ${ }^{1}$ Xiaomin Zhu, ${ }^{1}$ Jinlong Luo, ${ }^{1}$ and Baiyang $\mathrm{Ji}^{2}$ \\ ${ }^{1}$ Department of Mechanical Engineering, East China University of Science and Technology, Shanghai 200237, China \\ ${ }^{2}$ Hangzhou Sunyard System Engineering Co., Ltd., Hangzhou 310053, China \\ Correspondence should be addressed to Jianjun Yi; jjyi@ecust.edu.cn and Baiyang Ji; jby@sunyard.com
}

Received 5 November 2014; Revised 23 January 2015; Accepted 23 January 2015

Academic Editor: Marco Grassi

Copyright (c) 2015 Yujun Wang et al. This is an open access article distributed under the Creative Commons Attribution License, which permits unrestricted use, distribution, and reproduction in any medium, provided the original work is properly cited.

\begin{abstract}
Nowadays the cold chain logistics for perishable goods is increasingly complex, while most of the research works are focusing on the monitoring of temperature and humidity but seldom on the assessment and decision support for the monitored cold chain quality. In this context, a monitoring and decision system based on wireless sensor networks (WSN) and ontology is proposed in this paper which consists of sensing layer, network layer, and application layer. Ontology, as a shared concept model, can describe the objective world better with its own syntax and provides the general understanding of the specialized knowledge in a domain. Therefore, cold chain quality assessment software based on ontology has been developed; consequently, assessment and diagnosis for cold chain quality can be achieved, which can provide constructive advice and suggestions for its treatment. A demonstration of the system along a rabies vaccine logistics chain is validated in this paper. These results proved that this system presents important advantages such as effective regulation, low power consumption, and accurate ontology-based analysis.
\end{abstract}

\section{Introduction}

As people's living standard continues to rise, cold chain has achieved rapid development and wide attention. What is more, the quality and safety of food and drug is a top priority in relation to the national economy and people's livelihood. Consequently, achieving practical real-time monitoring is imminent.

Cold chain is complex, time-critical, and dynamic. It possesses several characteristics such as shelf life constraints for raw materials and perishability of products, long production throughput time, seasonality in production, necessity of conditioned transportation and storage, and safety concerns [1]. Most products such as food are perishable and their shelf life can be greatly affected by temperature and humidity conditions in the supply chain. Therefore, temperature and humidity control and timely management of goods are critical tasks for fresh food logistics [2]. Cold chain usually follows the principle of " $3 \mathrm{~T}$ "; it means that the product final quality depends on time, temperature, and tolerance [3]. Because of the accumulation and irreversibility of refrigerated product quality degradation in logistics, the logistics should be supervised strictly.

WSN, one of the ten essential technologies affecting people's lives in the 21st century [4], is a new emerging technique with respect to self-organized network, which combines the sensor technology, embedded computing technology, microelectromechanical system (MEMS) technology, modern network technology, and wireless communication technology [5]. WSN, bearing such characteristics as rapid expansion, strong antidisruptive capacity, and high fault tolerance, is widely used in the fields of military, aerospace, transportation, environment, and health [6]. Ontology, as a kind of knowledge representation, defines a set of representational primitives with which to model a domain of knowledge or discourse. The key role of ontologies with respect to database systems is to specify a data modeling representation at a level of abstraction above specific database designs (logical or physical), so that data can be exported, translated, 
queried, and unified across independently developed systems and services.

In our research, the temperature and humidity of each container, which is used to hold refrigerated goods in cold chain logistics car, should be guaranteed. Therefore, multiple cold chain tags are placed in the cold chain logistics car. In order to realize communication with coordinator simultaneously and quickly, WSN is used in this research. The WSN for cold chain logistics information system consists of coordinator and cold chain nodes. WSN-based system can get the temperature and humidity in real time, but it cannot give us the diagnosis, so ontology is also necessarily.

Based on WSN and ontology, a cold chain information system is proposed, which contains information collection system and cold chain quality assessment system, and the former, including cold chain tag and handheld terminal, is used for information collection, while the latter is used for cold chain quality evaluation and diagnosis and providing reasonable suggestions. Compared with traditional supervisory system, this system enjoys the following advantages:

(1) The low-power design of cold chain tag greatly reduces the energy consumption of the tag and significantly improves the service life of the tag.

(2) The hand-held terminal runs Windows Mobile operation system, and it cannot only display and store realtime data, but also transmit data wirelessly through GPRS. Besides, location of the cold chain tag can be shown on the GIS electronic map.

(3) The ontology-based system proposed in this paper can evaluate the cold chain quality with its detected parameters and provide reasonable suggestions.

The following parts of this paper are highlighted: Section 2 briefly introduces the cold-chain development, trend, and deficiency; Section 3 focuses on the overall design of the system; Section 4 expatiates the implementation of the system; Section 5 is validation of this system; and the final section is the conclusion.

\section{Literature Review}

ZigBee protocol is used in our WSN design. ZigBee is the set of specs built around the IEEE 802.15.4 wireless protocol. The IEEE is the Institute of Electrical and Electronics Engineers, a nonprofit organization dedicated to furthering technology involving electronics and electronic devices. While Bluetooth focuses on connectivity between large packet user devices, such as laptops, phones, and major peripherals, ZigBee is designed to provide highly efficient connectivity between small packet devices. The topology of ZigBee can be divided into the star topology, the tree topology, and the mesh topology.

The application of ZigBee technology in cold chain has been researched for years, especially in the field of Internet of Things (IoT), because of its low cost, low power consumption, short delay, and self-networking. Cold chain originated from the invention of the refrigerator in the middle of the 19th century. With the advent of refrigerators, a variety of fresh and frozen goods began to enter the market and consumer's family. After the 1930s, in Europe and the US, the cold chain system had been initially established. In 1894, Barrier and Ruddich firstly proposed the concept of cold chain. From the perspective of the study, the foreign research literature is quite shortage, and it only focuses on the stability, method assessment, and effect of cold chain.

Some scholars began to study the model optimization and management decisions of cold chain from a technical point and achieved some related analysis and forecast by mathematical statistical methods and BP artificial neural network technology [7]. In a cold chain management oriented design, an "Intelligent Container" was developed by Wang et al., and mesh topology of WSN was adopted to realize the multihop structure. The connectivity and stability were well maintained. The routing protocol of mesh topology belongs to the flat protocol. In a flat protocol network, all nodes should be functionally equal except the coordinator [8]. In another study, Sun and Liu [7] analyzed the current status of the fruit cold chain logistics in China, established the BP neural network model for the safety forewarning of fruit logistics. As a kind of knowledge representation, ontology has already achieved much progress and been applied in areas like medical diagnosis and medical design [9]. Recently, ontology is introduced to logistics. Xu et al. proposed a multiperspective ontologies' approach with intelligent agents. The multiperspective ontologies, involving static ontology, social ontology, and dynamic ontology, are firstly employed to develop the taxonomy of the logistics exception [10]. An ontology model specially designed for cold chain quality monitoring which could better represent and express the knowledge in cold chain quality monitoring domain does not exist. The aim of the ontology model built through the ontology-based knowledge modeling approach proposed in this paper is to provide a method for analyzing and assessing the data collected from the cold chain tag and giving recommended treatments in decision making process of cold chain logistics.

As mentioned above, the development of cold chain has attracted great attention of government, enterprises, and researchers. Such a good development environment shows the prosperity of cold chain future development. While, from the perspective of the researchers, existing regulatory systems and solutions are not satisfactory, the related research is significant and imminent.

\section{Framework of the Cold Chain Information System}

As shown in Figure 1, cold chain information system consists of three layers: sensing layer, network layer, and application layer.

Sensing layer is mainly used for data collection. The main device is the cold chain tag which consists of five parts: $\mathrm{MCU}$ module, sensor module, memory module, ZigBee module, and power module. CC2530 is chosen as the main control chip and SHT11 is used as temperature and humidity sensor. The sensor collects data once every ten minutes, and then the collected data is encapsulated according to protocol issued in 


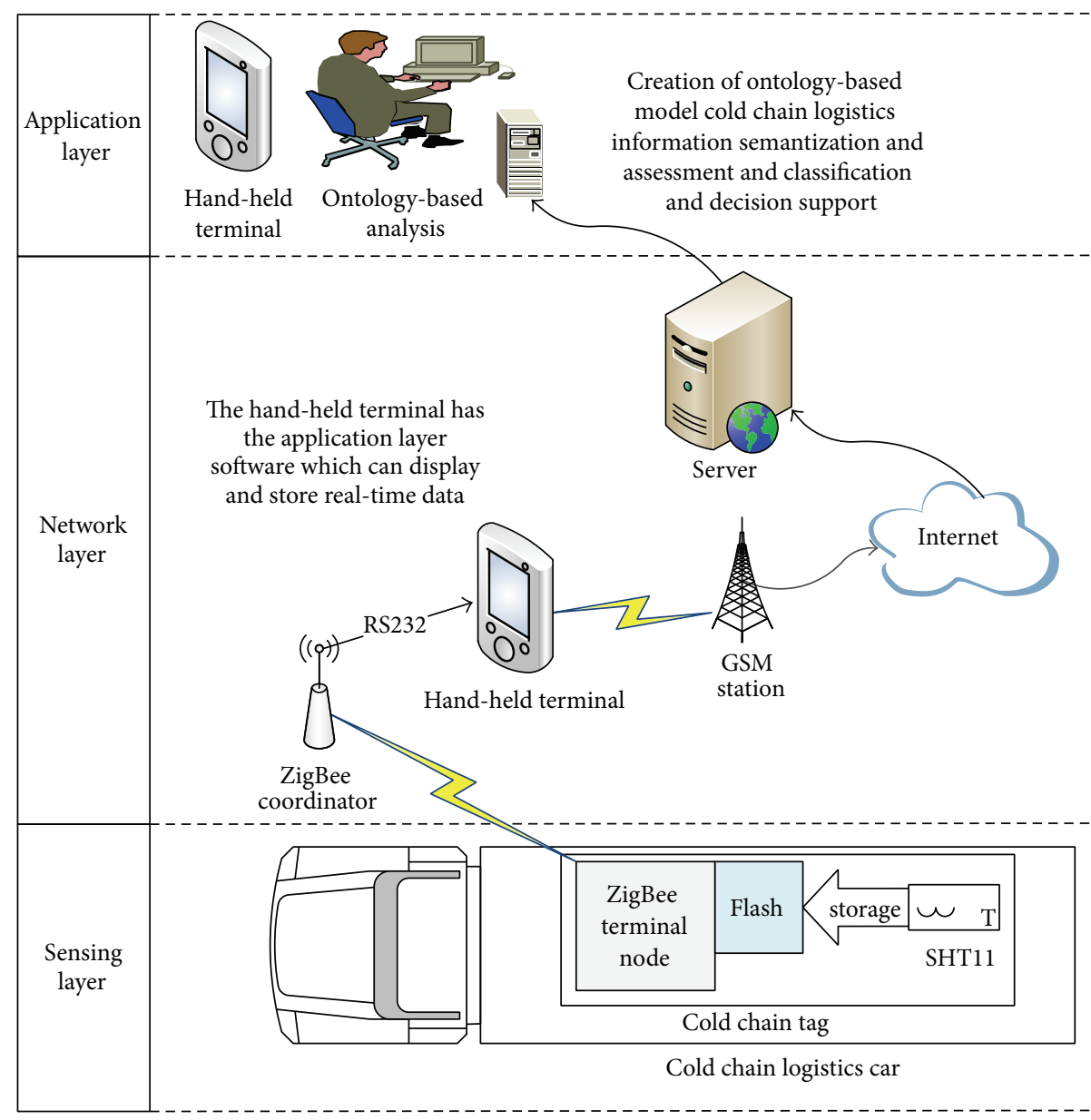

FIGURE 1: Framework of the food cold chain information system.

this paper. At last the processed data is saved in the flash of cold chain tag.

Network layer is responsible for sending data collected by sensing layer to application layer, which is the core technology of IoT. The ZigBee and GPRS wireless communication technology are mainly used in this research. The hand-held terminal is equipped with ZigBee coordinator node designed in this research, which can communicate with ZigBee terminal node and read the data saved in the cold chain tag flash. Then the hand-held terminal sends the data to remote server through GPRS for data storage and analysis. This is the whole data flow process in the network layer.

Application layer is mainly used for cold chain data storage, display, and analysis. In this paper, the application layer of cold chain information system consists of two subsystems: hand-held terminal information system and cold chain quality assessment system. The former mainly has three functions: the collection and processing of temperature and humidity data, the positioning and tracking of refrigerated goods, and system management. The latter system, established by ontology, is used for assessment and diagnosis of the cold chain quality. Coupled with a database in the remote server, an ontology-base model of cold chain quality information which is transformed into semantics one is created firstly, then a relatively complete assessment and classification on cold chain quality will be achieved, and corresponding suggestions or advices will be provided to supervision department.

\section{Implementation of the Cold Chain Information System}

As described above, the cold chain information system includes three layers. This paper mainly introduces the third layer (application layer).

4.1. Design of Hand-Held Terminal System Software. The hand-held terminal runs Windows Mobile operation system, coupled with a 3.5-inch touch screen, GPS module, GPRS module, large volume battery, and connector connecting to ZigBee module. Communication between hand-held terminal and ZigBee coordinator is realized through a serial port, and the terminal supplies needed power for ZigBee coordinator. 


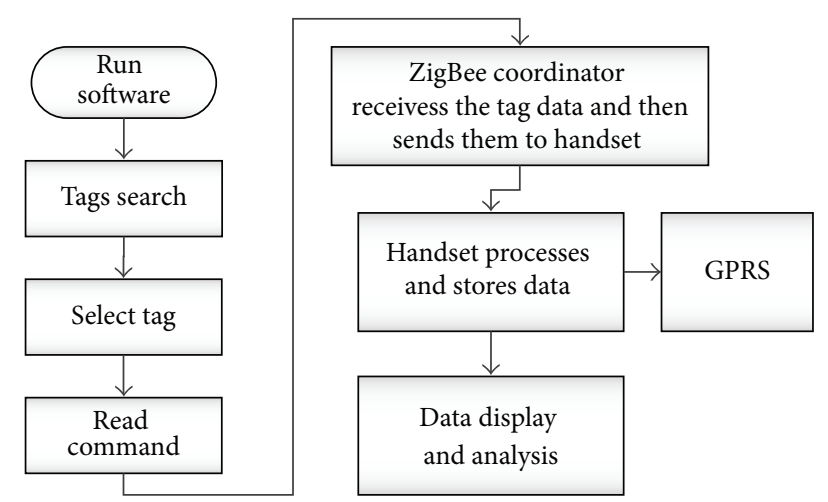

FIGURE 2: Software running flow chart.

The software is mainly used for reading, storing, processing, displaying, and sending the temperature and humidity data of cold chain tag. In order to enter the system, users need to $\log$ in at first. The software sends command to cold chain tag through ZigBee coordinator. Then the cold chain tag will return answer data after receiving the command; the answer data will be sent to hand-held terminal through coordinator. At last, the hand-held terminal sends the data to remote server for cold chain quality assessment, The workflow is shown in Figure 2.

The software, developed on the WinCE platform with program language $\mathrm{C} \#$, has friendly user interfaces. It consists of five functional modules: system management, data entry, data upload, navigation, and data query. Users can use it to complete data processing and displaying and realize tracking by GPS and GIS. The software architecture is shown in Figure 3.

(1) System Management. Only administrator has the right to operate this function module. It consists of user management and system setting. Here, users can modify the user information and system information.

(2) Data Entry. In this research, there are three ways of data entry: read all, read by package, and read by time. The temperature and humidity data is stored in cold chain tag in groups, 17 data as a group. The mode of "read all" is reading all data in the flash; "read by time" is reading the data near given time; "read by group" is reading the given group data. The three modes are combined with each other, which greatly improves the flexibility and usability of the system.

(3) Data Upload. This function is used for sending the data stored in hand-held terminal to remote server via GPRS.

(4) Navigation. The software also has GIS and GPS functions, which help to position and track the cold chain tag accurately.

(5) Data Query. Users can use this function to query historical data, such as temperature and humidity data and user information.

4.2. Implementation of Ontology-Based Cold Chain Quality Assessment System. A complete cold chain information system not only needs to achieve cold chain information collection, acceptance, and display but also needs to complete cold chain process quality assessment and diagnosis and then provides a reference to the relevant departments. Cold chain process has characteristics of parameter diversity and control indicators variability; it is difficult for traditional analytical models to achieve the desired quality assessment results. This research makes use of ontology to semantically process the cold chain information, such as temperature and humidity information, location information, time information, and refrigerated product information. Then an ontology model which will read information from the database is established in this research. At last, the ontology-based instance is created. On this basis, rules of inference are established, and then inference engine based on inference rules can give out the final result. As shown in Figure 4, it is the development flow of cold chain quality assessment system based on ontology.

4.2.1. Establishment of Ontology-Based Cold Chain Quality Information Model. The cold chain quality ontology proposed in this paper is mainly used in the field of cold chain; thus it can be classified as highly specialized domain ontology. Currently, no method has passed the certification authority for establishing domain ontology. Such methods as TOVE, METHONTOLOGY, Skeleton Method, KACTUS, SENSUS, IDEF5, and Seven-Step Method can be applied for domain ontology building. And the Seven-Step Method, developed by the Medical Information Center of Stanford University [11, 12], is among top choices to build domain ontology. Besides, as it enjoys detailed technical support and advantages in model creation, the Seven-Step Method is adopted in this research.

This research selects Protégé [13] as the modeling tool to describe the ontology knowledge. Protégé is a free, opensource ontology editor and framework for building intelligent systems. It is an extensible tool based on Java and provides a plug-and-play environment that makes it a flexible base for rapid prototyping and application development [14]. As shown in Figure 5, the ontology model of cold chain information mainly consists of diagnosis class and parameter class. In the parameter class, there are five subclasses. For the subclasses "temperature" and "humidity," both of them have two instances, which represents the upper and lower limit of temperature and humidity in the clod chain logistics. Users could change the values of the two instances to adapt the ontology model for different kinds of cold chain applications. The subclass "location" has instances including "starting point," "middle point," and "end point," which represent the three important locations in the logistics process. The subclass "time" includes the important time points in the logistics including the time at starting point and end point. The subclass "product information" has instances including "name," "manufacturers," "type," and "storage," which is used to represent the product information. In the class "diagnosis," there are five instances including "normal," "Alarm I," "Alarm II," "Alarm III," and "Alarm IV." They represent the diagnosis result of the cold chain logistics process. 


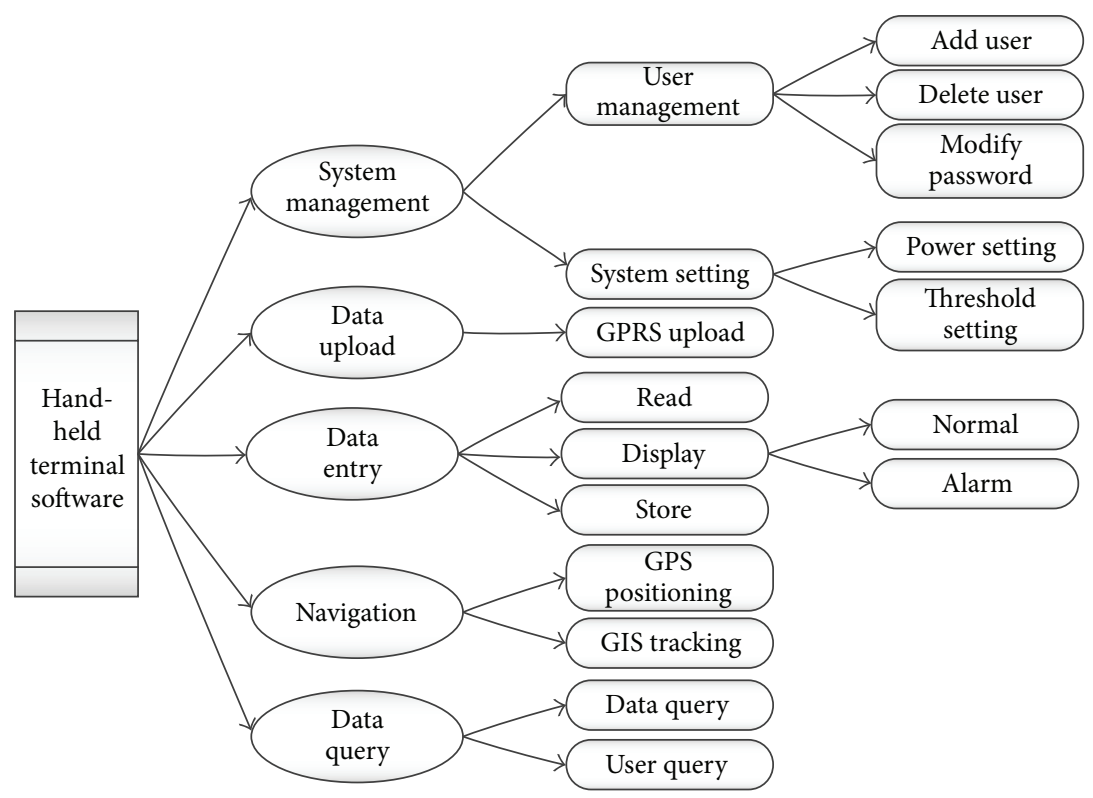

FIGURE 3: Hand-held terminal software architecture diagram.

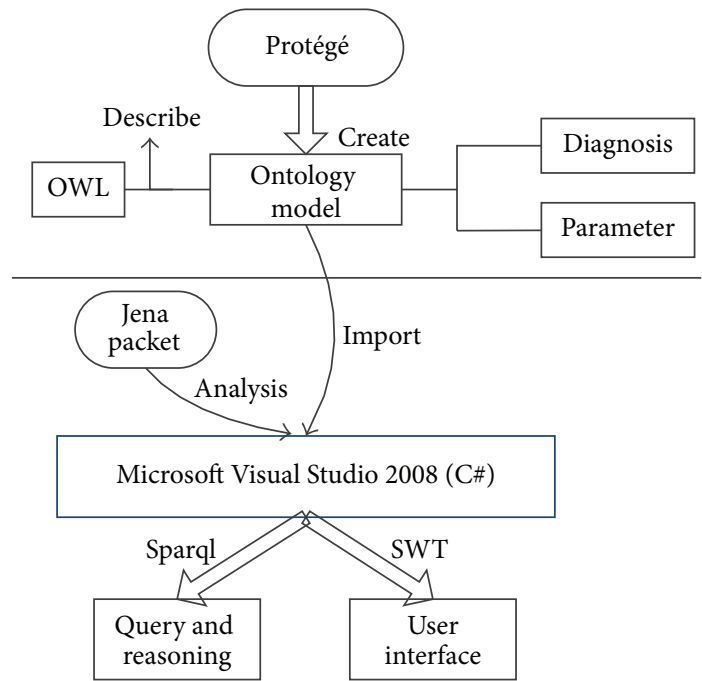

FIGURE 4: Establishment process of food cold chain quality assessment system.

After building the cold chain quality ontology model, the validity of ontology organizational structure and class property definition still need to be checked. This research uses Pellet 1.5.2 to test it, and no abnormal situation occurred in the testing process. Therefore, the ontology model is correct and reasonable.

4.2.2. Implementation of Cold Chain Information Ontology Analysis. Construction of cold chain information ontology model described earlier is the precondition of cold chain ontology analysis. Besides, this research also needs to develop related application to analyze and operate the ontology model. At last, intelligent inference will be achieved by making full use of the created constraints, and decision support for managers will be provided. The application software is developed by C\#. The process of its implementation is that since Protégé uses Java language development, Java classes and Jena [15] package should be added to the platform of Visual Studio 2008 firstly. Secondly, import the cold chain information ontology into the development environment through the C\# programming mechanism. Thirdly, inquire the information of assessment index through Sparql language. Fourthly, use comprehensive assessment method to assess the cold chain information. Finally, use Jena inference engine to analyze the status of refrigerated goods and give recommendations. Users can get the result from the software interface intuitively.

(1) Analysis of Cold Chain Quality Ontology Based on Jena. Jena is an open source Java software for developing Semantic Web applications. Jena framework includes an ontology subsystem, supporting OWL, DAML + OIL, and RDFS. This research mainly uses the query model in Jena API and rule-based inference. The former extracts information from ontology model by "com.hp.hpl.jena.ontology" package and the latter achieves the Jena inference of cold chain quality ontology by "com.hp.hpl.jena.reasoner" package.

The analysis of cold chain quality ontology by Jena API in Visual Studio 2008 development platform mainly includes the following three steps: Firstly, import Jena and Java package into the application for software development and include the required namespace in the application. Secondly, use "createOntologyModel()" to instantiate the ontology model. Finally, read the .owl document by file stream.

So far, the reading and analysis of cold chain quality ontology is achieved. Users can inquire the information in ontology by Sparql and achieve inference by Jena. 


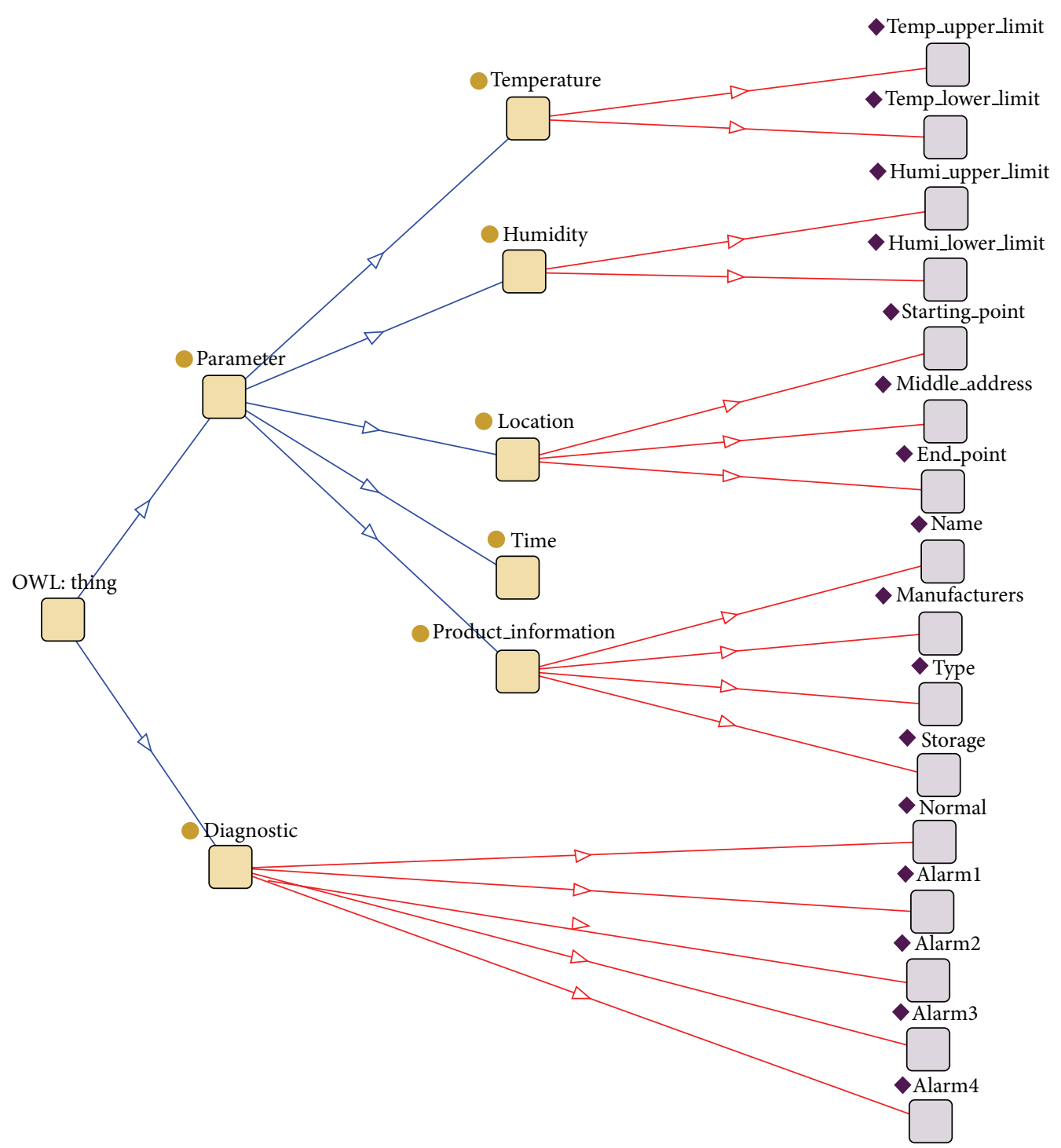

FIGURE 5: Semantic processing cold chain information ontology model.

(2) Implementation of Query Inference Based on Sparql and Jena. Sparql [16] (Simple Protocol and RDF Query Language) is a query language and data acquisition protocol for RDF, but it only can inquire about the information existing in model without inference function. Therefore, Sparql is mainly used for reading data for application by inquiry. In this research, the query result stores in the corresponding .txt file, and application extracts available information from the file according to request.

Sparql mainly has four query forms: they are SELECT, CONSTRUCE, ASK, and DESCRIBE. This research uses SELECT to query the assessment index criteria of cold chain quality ontology. After getting the information, application will compare it with acquired parameter value and then get real-time status of the cold chain quality. The technical route is shown in Figure 6.

\section{Validation of Cold Chain Information System}

The cold chain quality assessment system can work properly only when all modules (data acquisition module, data transmission and data management module, and data analysis module) of the cold chain information system work well. Therefore, the system test has been undertaken and results show high efficiency and reliability achieved, as all modules in this system work smoothly.

Test Content. In consideration of the feasibility of test, this research takes the transport of rabies vaccine from Xuhui district central hospital to Zhabei district central hospital as an example. The temperature and humidity data collected by sensors will be sent to the hand-held terminal through ZigBee 


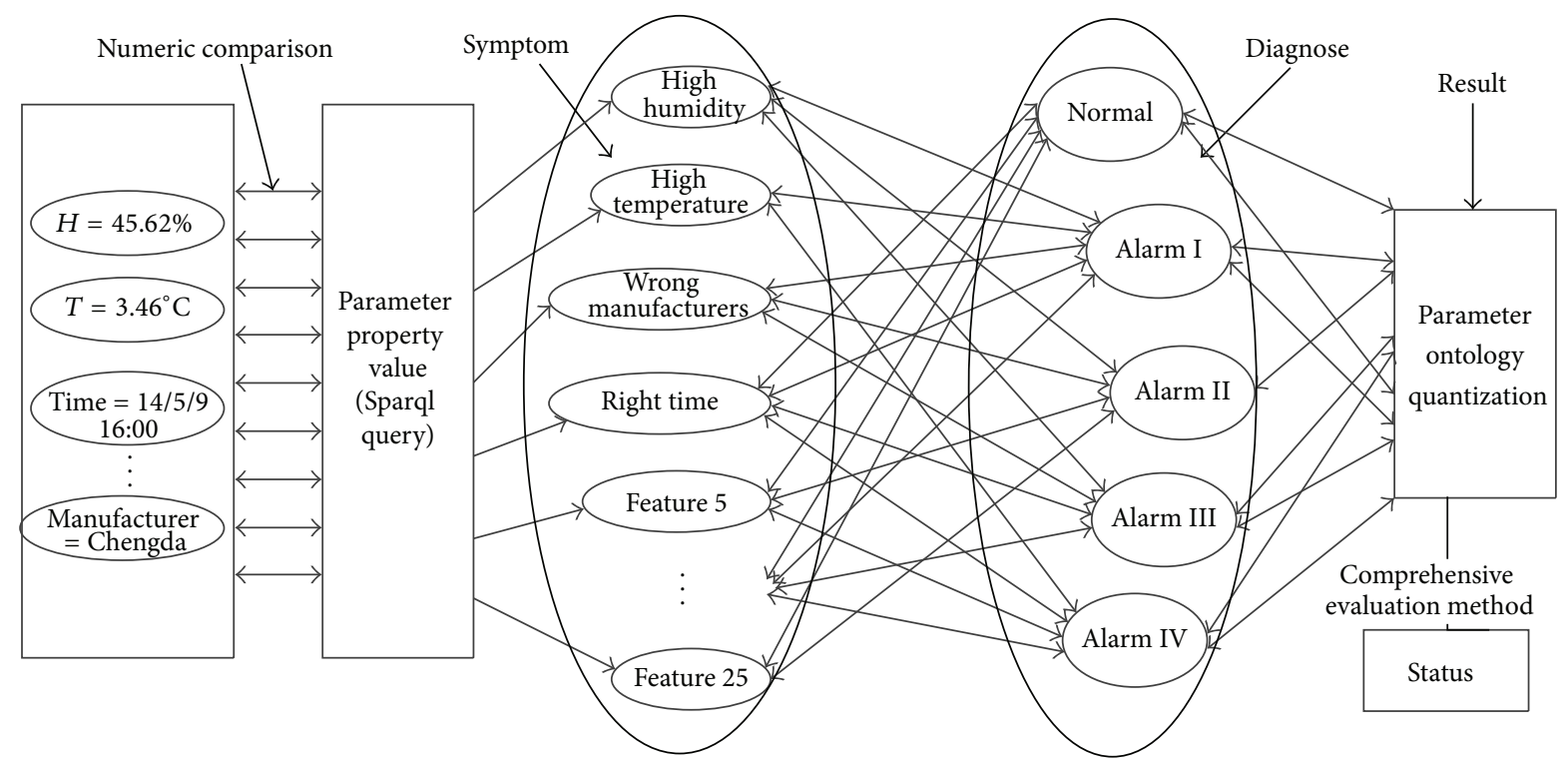

FIGURE 6: Sparql query technical route.

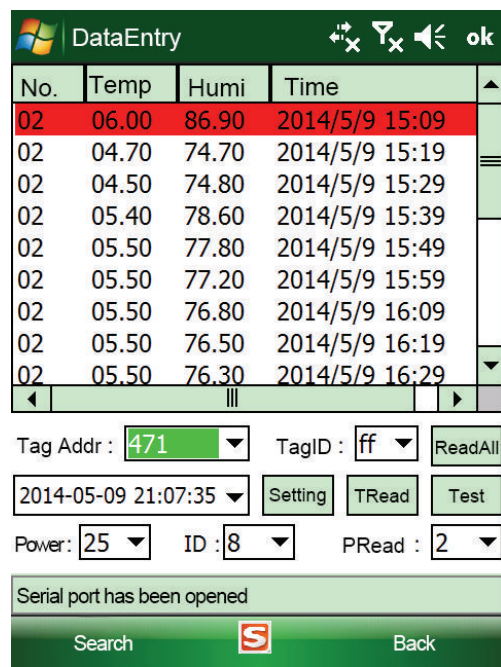

(a)

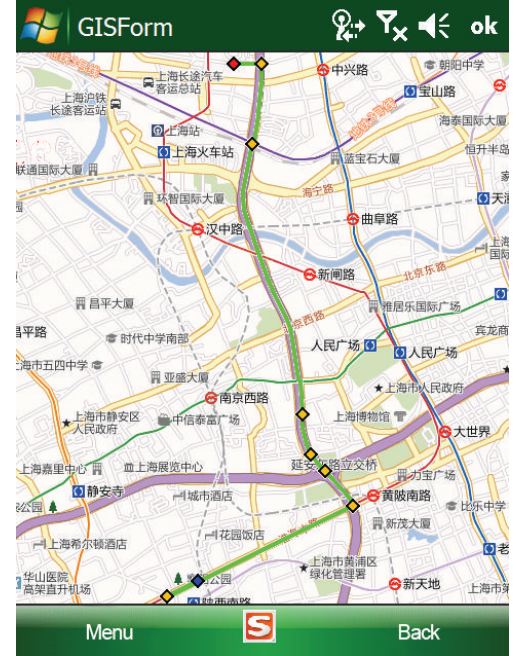

(b)

FIgURE 7: (a) Acquisition of temperature and humidity data. (b) Acquisition of geographic information.

module after preliminary processing, and then it will be sent to remote monitoring center. In the remote monitoring center, the application, developed in this research, stores the data in database as the original data of cold chain information diagnosis system. The process of information collection is shown in Figure 7. Combining with the time and product information, the results got by the cold chain assessment system are shown in Table 1, which is consistent with the result got from reference information. In conclusion, with all modules working smoothly, the cold chain quality assessment system based on ontology can make accurate evaluation on the cold chain quality and operates steadily, therefore, meeting the requirements of cold chain quality information analysis.

\section{Conclusions}

In this paper, the design and implementation of cold chain information system are discussed and an ontology-based assessment system is established. This ontology-based system has realized intelligent reasoning on cold chain quality; thus it can give out assessment results of cold chain quality. Users can acquire accurate diagnostic information more directly. It also improves the transparency of the cold chain process and ensures the effectiveness of supervision. The cold chain information system proposed in the paper, because of the simple operation, flexible application, and high stability, can be widely used in the logistics monitoring of refrigerated goods, such as fruit, vegetables, and vaccine. 
TABLE 1: Monitoring parameter values and ontology diagnosis.

\begin{tabular}{|c|c|c|c|c|c|}
\hline Parameter & 1st group & 2nd group & 3rd group & 4th group & 5th group \\
\hline Temperature & -2.30 & 0.20 & 2.60 & 9.20 & 5.50 \\
\hline Humidity & 70.20 & 85.10 & 88.20 & 91.70 & 77.20 \\
\hline Start & XuHui & XuHui & XuHui & XuHui & XuHui \\
\hline End & ZhaBei & ZhaBei & ZhaBei & ZhaBei & ZhaBei \\
\hline Mid-address & JingAn & JingAn & JingAn & JingAn & JingAn \\
\hline Time & $14 / 4 / 922: 09$ & 14/4/14 11:39 & $14 / 4 / 2513: 19$ & $14 / 4 / 3010: 49$ & $14 / 15 / 9$ 15:09 \\
\hline Storage temp. & 5 & 5 & 5 & 5 & 5 \\
\hline Product name & Vaccine & Vaccine & Vaccine & Vaccine & Vaccine \\
\hline Type & Injection & Injection & Injection & Injection & Injection \\
\hline Number & 10 & 10 & 10 & 10 & 10 \\
\hline Manufacturer & Chengda & Chengda & Chengda & Chengda & Chengda \\
\hline Conveyance & Car & Car & Car & Car & Car \\
\hline Validity period & $15 / 4 / 30$ & $14 / 8 / 30$ & $14 / 12 / 30$ & $15 / 6 / 30$ & $15 / 10 / 30$ \\
\hline Results & Alarm IV & Alarm III & Alarm II & Alarm I & Normal \\
\hline
\end{tabular}

\section{Conflict of Interests}

The authors declare that there is no conflict of interests regarding the publication of this paper.

\section{Acknowledgments}

This paper is supported by the Research Foundation of Science and Technology Commission of Shanghai under Grant no. 10DZ1500200, the Nature Science Fund of China (NSFC) under Grant nos. 50975088, 51275173, and 51210105018, the Fundamental Research Funds for the Central Universities under Grant no. WH0913009, Shanghai Pujiang Program under Grant no. PJ201000353, and Shanghai Software and IC industry Development Special Fund under Grant no. 120493.

\section{References}

[1] L. H. Aramyan, A. G. J. M. O. Lansink, J. G. A. J. van der Vorst, and O. V. Kooten, "Performance measurement in agrifood supply chains: a case study," Supply Chain Management, vol. 12, no. 4, pp. 304-315, 2007.

[2] M. M. Aung and Y. S. Chang, "Temperature management for the quality assurance of a perishable food supply chain," Food Control, vol. 40, no. 1, pp. 198-207, 2014.

[3] C. Huang and R. Xie, "Development and countermeasure of food cold chain logistics in China," Storage, Transportation \& Preservation of Commodities, vol. 29, no. 4, pp. 37-39, 2007.

[4] Z. Jing, S. Gang, Z. Chimin, and W. Jie, "Research and application of wireless sensor networks in water quality monitor," Communications Technology, vol. 41, no. 4, pp. 124-126, 2008.

[5] F. Libo, Y. Honglan, and Z. Xinping, "The design and realization of farmland information monitoring system based on LabVIEW," Journal Of Anhui Agricultural Sciences, vol. 39, no. 9, pp. 5563-5565, 2011.

[6] M. F. Othman and K. Shazali, "Wireless sensor network applications: a study in environment monitoring system," Procedia Engineering, vol. 41, no. 1, pp. 1204-1210, 2012.
[7] J. Sun and H. Liu, "Study on fruit cold-chain logistics forewarning model based on BP neural network," Logistics Technology, vol. 32, no. 5, pp. 204-207, 2013.

[8] J. Wang, H. Wang, J. He et al., "Wireless sensor network for realtime perishable food supply chain management," Computers and Electronics in Agriculture, vol. 110, pp. 196-207, 2015.

[9] F. N. Natalya and L. M. Deborah, "Ontology Development 101: A Guide to Creating Your First Ontology," 2000, http://protege.stanford.edu/publications/ontology_development/ontology101.pdf.

[10] D. Xu, C. Wijesooriya, Y.-G. Wang, and G. Beydoun, "Outbound logistics exception monitoring: a multi-perspective ontologies' approach with intelligent agents," Expert Systems with Applications, vol. 38, no. 11, pp. 13604-13611, 2011.

[11] A. Scheuermann and J. Leukel, "Supply chain management ontology from an ontology engineering perspective," Computers in Industry, vol. 65, no. 6, pp. 913-923, 2014.

[12] Z. Qinglei, "Analysis on the laws and rules of urban lake water management," Cards World, vol. 15, no. 6, pp. 1-3, 2011.

[13] I. Smeureanu and B. Iancu, "Source code plagiarism detection method using Protégé built ontologies," Informatica Economica, vol. 17, no. 3, pp. 75-86, 2013.

[14] C. P. Alderfer, "Clarifying the meaning of mentor-protégé relationships," Consulting Psychology Journal, vol. 66, no. 1, pp. 6-19, 2014.

[15] J. Wu and Y. Huang, "Reasoning with ontology model based on Jena," Intelligent Information Management, vol. 1, no. 1, pp. 2429, 2009.

[16] S. Shekarpour, S. Auer, A.-C. N. Ngomo, D. Gerber, S. Hellmann, and C. Stadler, "Generating SPARQL queries using templates," Web Intelligence and Agent Systems, vol. 11, no. 3, pp. 283-295, 2013. 

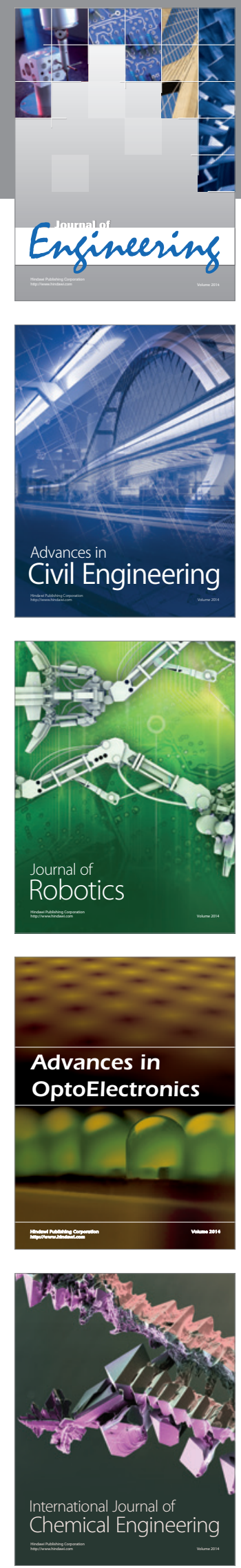

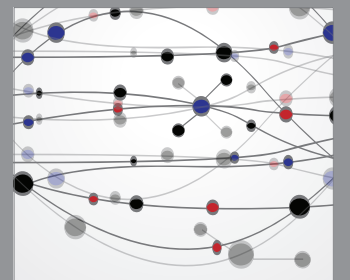

The Scientific World Journal
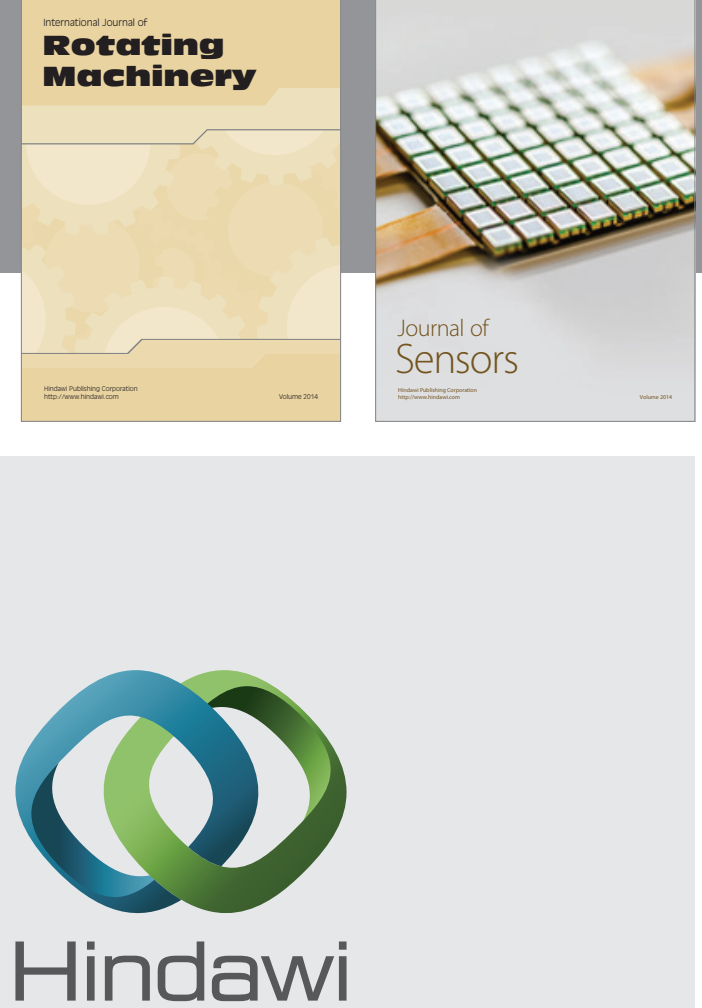

Submit your manuscripts at http://www.hindawi.com
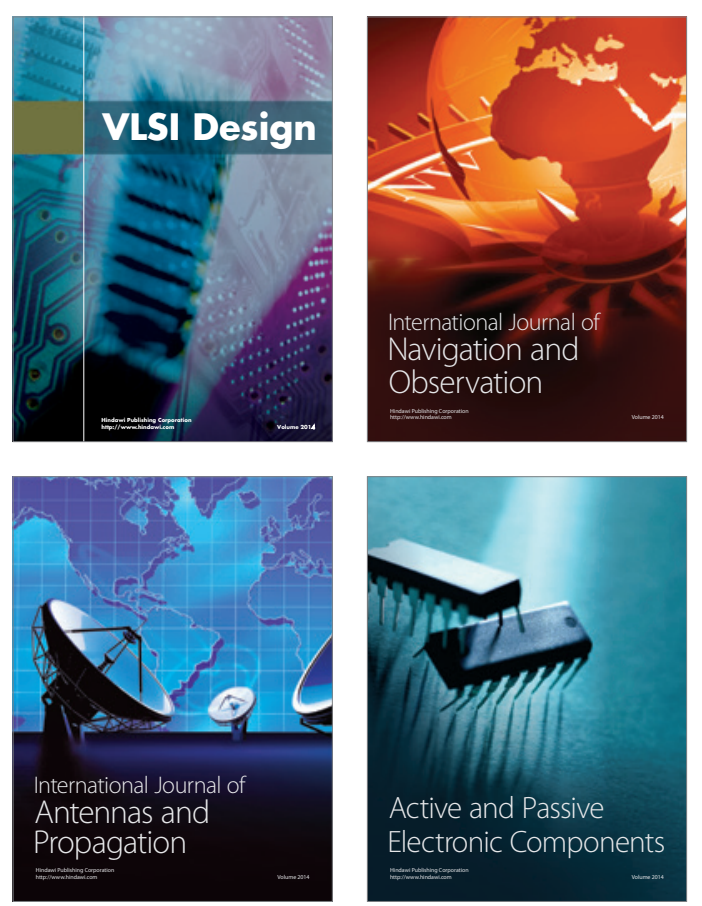
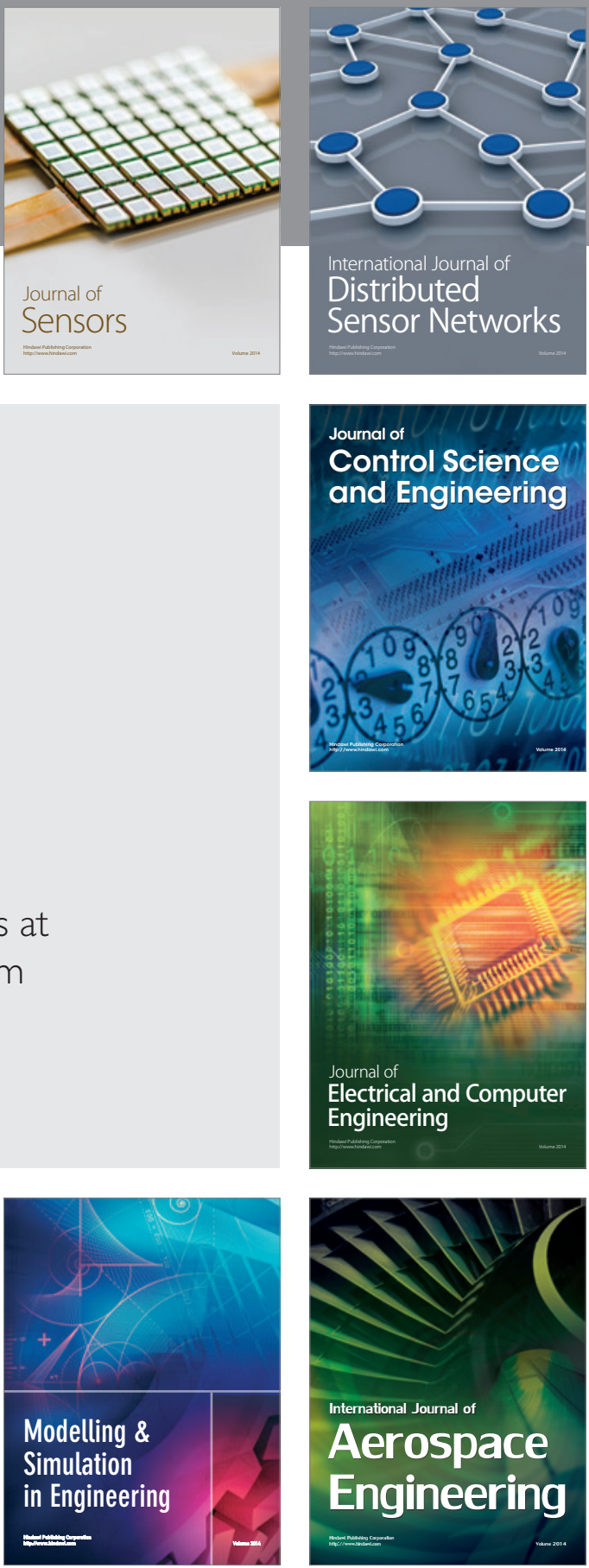

Journal of

Control Science

and Engineering
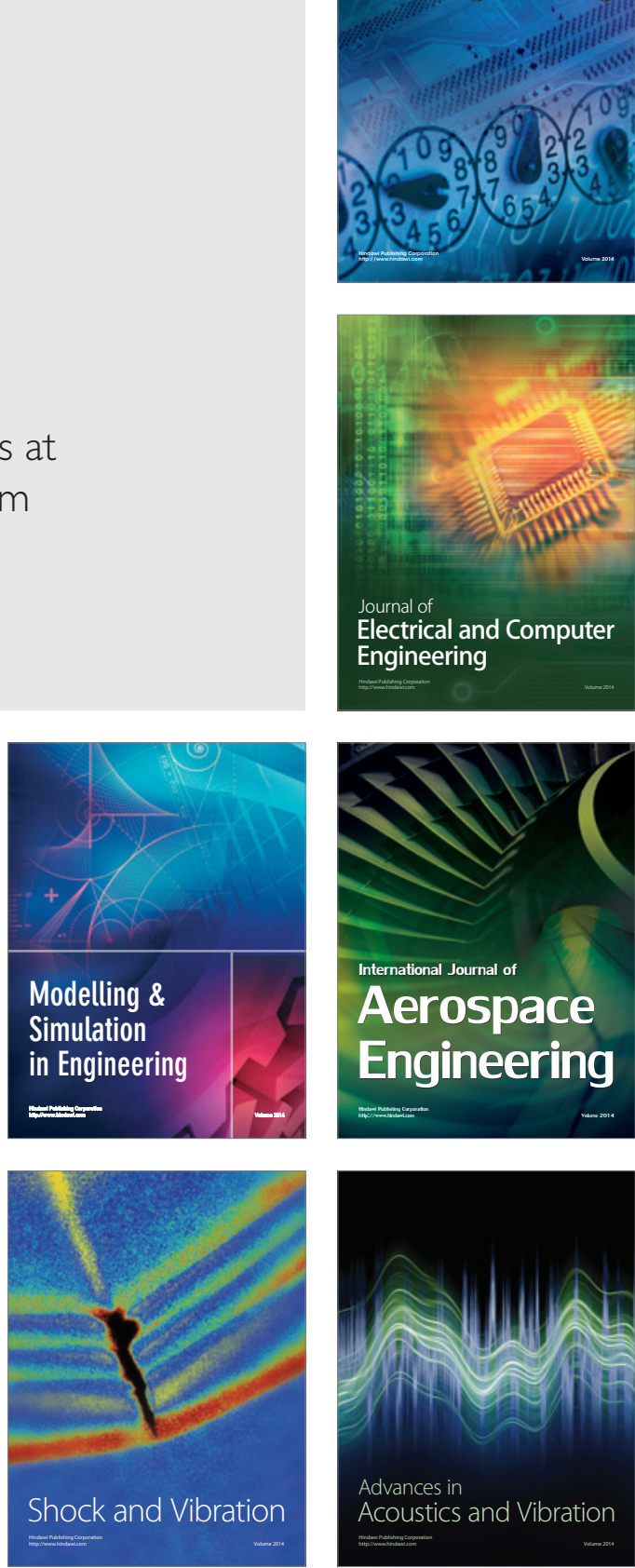